\title{
Use of Minerals and Materials in the United States From 1900 Through 2006
}

Since the beginning of the 20th century, the types and quantities of raw materials used by U.S. manufacturers and consumers have changed significantly as the economy has moved from one that is predominantly agricultural to one that is primarily industrial. This fact sheet quantifies the materials (other than food and fuel) input into the U.S. economy from 1900 through 2006.

Previous studies have tracked the flow of minerals and materials by monetary value as a way of determining their effect on the U.S. economy. This information is the first attempt to document the flow of these materials in physical terms, which can help in assessing their potential adverse effect on the physical environment. This fact sheet presents the amounts (by weight) of raw minerals and materials used in the four categories of physical goods that support the U.S. economy. These four categories are agriculture, forestry, metals and minerals, and nonrenewable organics.

Table 1 is a listing of the amount of minerals and materials used, and figures 1 and 2 are graphical representations of these data. Figure 3 is a listing of those minerals and materials used in the United States from 1900 through 2006. Note that the data in the table are only for materials that are ready for use or manufacture in products that were actually made in the United States, not for materials contained in finished goods that were imported into or exported out of the country. These data represent the annual apparent inputs to the use phase (domestic production plus imports plus recycling minus exports) aggregated by category. For example, even though a significant amount of cadmium metal is contained in electronic goods imported into the country, the cadmium data provided in this study are only for the cadmium used in products that were produced in the United States. In an industrial economy such as that of the United States, where the volume of goods flowing into and out of the country is large, tracking the flow of materials embedded in imported products is not only difficult but virtually impossible. The data provided are for the first point of consumption; ultimate consumption in the form of manufactured products could be significantly different.

Throughout the lifecycle of any material, the potential exists for losses to and adverse effects on

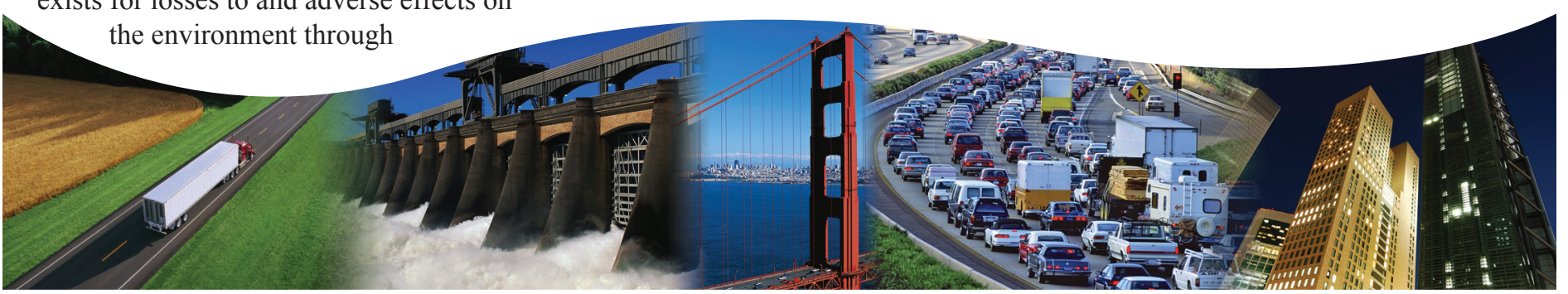

emissions, losses, wastes, or dissipation of materials into the environment.

Many sources were used to compile the data for this fact sheet. The following is a list of the primary ones used:

- U.S. Department of Agriculture, Agricultural Statistics (for agriculture statistics)

- National Oceanic and Atmospheric Administration, Fisheries of the United States (for fisheries statistics)

- U.S. Bureau of Mines and U.S. Geological Survey, Mineral Resources of the United States, and Minerals Yearbook (for metal and mineral statistics)

- U.S. Census Bureau, Statistical Abstract of the United States (for agriculture statistics)

- Resources for the Future, Natural Resource Commodities-A Century of Statistics (for agriculture statistics)

- U.S. Energy Information Administration, Annual Energy Review (for nonrenewable organics statistics)

- U.S. Forest Service, U.S. Timber Production, Trade, Consumption, and Price Statistics (for forestry and paper statistics)

- U.S. International Trade Commission, Synthetic Organic Chemicals (for nonrenewable organics statistics, and statistics on primary products made from petroleum and natural gas)

\section{By Grecia R. Matos}

\section{For more information, contact:}

Minerals Information Team U.S. Geological Survey

Reston, VA 20192

http://minerals.usgs.gov/minerals 
Table 1. Raw materials used in the United States from 1900 through 2006.

[In thousand metric tons. --, negligible or no data]

\begin{tabular}{|c|c|c|c|c|c|c|c|c|c|c|}
\hline \multirow[b]{2}{*}{ Year } & \multirow[b]{2}{*}{ Agriculture } & \multicolumn{3}{|c|}{ Forestry } & \multicolumn{4}{|c|}{ Metals and minerals } & \multirow[b]{2}{*}{$\begin{array}{c}\text { Nonrenewable } \\
\text { organics }\end{array}$} & \multirow[b]{2}{*}{$\begin{array}{c}\text { Total of } \\
\text { materials }\end{array}$} \\
\hline & & Wood & $\begin{array}{l}\text { Paper and } \\
\text { paperboard }\end{array}$ & $\begin{array}{c}\text { Recycled } \\
\text { paper }\end{array}$ & $\begin{array}{c}\text { Primary } \\
\text { metals }\end{array}$ & $\begin{array}{c}\text { Recycled } \\
\text { metals }\end{array}$ & $\begin{array}{l}\text { Industrial } \\
\text { minerals }\end{array}$ & $\begin{array}{c}\text { Construction } \\
\text { materials }\end{array}$ & & \\
\hline 1900 & 3,040 & 60,300 & 2,640 & -- & 10,300 & -- & 10,800 & 55,300 & 1,590 & 144,000 \\
\hline 1901 & 3,460 & 62,800 & 2,670 & -- & 13,100 & -- & 11,300 & 61,000 & 1,670 & 156,000 \\
\hline 1902 & 3,660 & 65,600 & 2,690 & -- & 14,800 & -- & 13,300 & 70,000 & 1,760 & 172,000 \\
\hline 1903 & 3,300 & 67,100 & 2,720 & -- & 14,100 & -- & 14,000 & 67,000 & 1,880 & 170,000 \\
\hline 1904 & 3,560 & 69,000 & 2,750 & -- & 12,900 & -- & 20,100 & 70,100 & 1,900 & 180,000 \\
\hline 1905 & 3,680 & 70,600 & 2,720 & -- & 17,800 & -- & 23,000 & 81,200 & 1,960 & 201,000 \\
\hline 1906 & 3,740 & 75,800 & 2,690 & -- & 20,800 & 12 & 27,400 & 95,200 & 2,040 & 228,000 \\
\hline 1907 & 3,310 & 80,800 & 2,670 & -- & 21,100 & 44 & 28,700 & 90,000 & 2,130 & 229,000 \\
\hline 1908 & 3,570 & 74,200 & 2,640 & -- & 12,900 & 29 & 25,400 & 81,900 & 2,170 & 203,000 \\
\hline 1909 & 3,490 & 78,800 & 3,720 & -- & 20,900 & 83 & 31,000 & 109,000 & 2,260 & 249,000 \\
\hline 1910 & 3,380 & 78,800 & 3,680 & -- & 23,000 & 123 & 34,900 & 125,000 & 2,370 & 271,000 \\
\hline 1911 & 3,710 & 75,900 & 3,650 & -- & 20,400 & 133 & 35,500 & 122,000 & 2,590 & 264,000 \\
\hline 1912 & 4,090 & 77,700 & 3,610 & -- & 26,200 & 184 & 38,300 & 124,000 & 2,930 & 277,000 \\
\hline 1913 & 3,800 & 76,200 & 3,570 & -- & 26,400 & 180 & 39,300 & 141,000 & 3,310 & 294,000 \\
\hline 1914 & 3,890 & 72,400 & 4,890 & -- & 18,800 & 152 & 37,300 & 130,000 & 3,560 & 271,000 \\
\hline 1915 & 4,000 & 69,300 & 4,840 & -- & 23,200 & 219 & 38,800 & 131,000 & 4,230 & 276,000 \\
\hline 1916 & 4,140 & 73,000 & 4,800 & -- & 29,700 & 295 & 43,800 & 132,000 & 4,870 & 293,000 \\
\hline 1917 & 4,120 & 69,200 & 5,490 & -- & 30,500 & 297 & 45,400 & 114,000 & 5,360 & 274,000 \\
\hline 1918 & 4,050 & 65,200 & 5,690 & -- & 29,200 & 277 & 40,600 & 93,300 & 5,250 & 244,000 \\
\hline 1919 & 3,860 & 67,400 & 5,670 & -- & 23,300 & 290 & 38,900 & 97,600 & 5,530 & 243,000 \\
\hline 1920 & 3,750 & 67,200 & 6,900 & -- & 30,300 & 307 & 48,200 & 115,000 & 5,790 & 277,000 \\
\hline 1921 & 3,520 & 58,200 & 5,470 & -- & 14,100 & 243 & 36,500 & 104,000 & 5,160 & 228,000 \\
\hline 1922 & 4,170 & 64,100 & 7,130 & -- & 26,700 & 382 & 47,800 & 125,000 & 6,380 & 282,000 \\
\hline 1923 & 4,220 & 70,100 & 8,340 & -- & 34,100 & 486 & 57,900 & 173,000 & 8,390 & 357,000 \\
\hline 1924 & 4,260 & 67,200 & 8,420 & -- & 29,000 & 495 & 59,500 & 194,000 & 10,000 & 373,000 \\
\hline 1925 & 4,560 & 66,300 & 9,450 & -- & 34,500 & 558 & 65,900 & 214,000 & 10,400 & 406,000 \\
\hline 1926 & 4,600 & 65,100 & 10,500 & -- & 36,600 & 653 & 67,100 & 232,000 & 10,600 & 427,000 \\
\hline 1927 & 4,860 & 61,300 & 10,800 & -- & 33,800 & 653 & 66,000 & 255,000 & 11,200 & 444,000 \\
\hline 1928 & 4,750 & 59,500 & 11,300 & -- & 38,400 & 715 & 68,200 & 249,000 & 12,900 & 444,000 \\
\hline 1929 & 4,930 & 61,700 & 12,200 & -- & 42,000 & 750 & 69,600 & 282,000 & 14,400 & 487,000 \\
\hline 1930 & 4,410 & 49,500 & 11,200 & -- & 30,600 & 619 & 60,000 & 255,000 & 13,900 & 425,000 \\
\hline 1931 & 4,280 & 36,200 & 10,300 & -- & 20,300 & 506 & 46,800 & 193,000 & 12,300 & 324,000 \\
\hline 1932 & 3,640 & 27,500 & 8,830 & -- & 11,300 & 387 & 32,000 & 149,000 & 10,600 & 243,000 \\
\hline 1933 & 4,180 & 31,200 & 9,900 & -- & 17,500 & 506 & 33,100 & 143,000 & 11,100 & 250,000 \\
\hline 1934 & 3,620 & 32,900 & 10,200 & -- & 19,400 & 540 & 37,100 & 168,000 & 12,500 & 284,000 \\
\hline 1935 & 4,070 & 38,000 & 11,600 & -- & 25,000 & 657 & 42,100 & 165,000 & 13,200 & 300,000 \\
\hline 1936 & 4,360 & 44,500 & 13,300 & -- & 35,500 & 682 & 54,500 & 249,000 & 15,900 & 418,000 \\
\hline 1937 & 5,010 & 46,800 & 14,500 & -- & 39,200 & 739 & 59,800 & 259,000 & 17,400 & 442,000 \\
\hline 1938 & 4,040 & 41,300 & 12,300 & -- & 25,000 & 520 & 48,300 & 250,000 & 17,200 & 398,000 \\
\hline 1939 & 5,280 & 46,000 & 14,500 & -- & 39,300 & 573 & 58,600 & 304,000 & 18,900 & 487,000 \\
\hline 1940 & 6,420 & 48,700 & 15,200 & -- & 44,900 & 660 & 65,000 & 319,000 & 19,200 & 519,000 \\
\hline 1941 & 7,280 & 56,300 & 18,500 & -- & 61,200 & 922 & 83,900 & 380,000 & 22,400 & 631,000 \\
\hline 1942 & 6,410 & 56,300 & 17,900 & -- & 59,300 & 936 & 89,200 & 402,000 & 22,300 & 655,000 \\
\hline 1943 & 6,640 & 52,100 & 17,600 & -- & 59,200 & 1,040 & 95,600 & 325,000 & 21,700 & 579,000 \\
\hline 1944 & 6,260 & 50,400 & 17,600 & -- & 59,000 & 1,090 & 88,500 & 281,000 & 23,100 & 527,000 \\
\hline 1945 & 6,070 & 44,500 & 17,800 & -- & 56,400 & 1,090 & 89,800 & 279,000 & 25,600 & 520,000 \\
\hline 1946 & 6,800 & 51,400 & 20,400 & -- & 48,600 & 1,020 & 107,000 & 353,000 & 27,500 & 616,000 \\
\hline 1947 & 6,800 & 53,000 & 22,400 & -- & 60,500 & 1,280 & 131,000 & 394,000 & 28,900 & 698,000 \\
\hline 1948 & 7,310 & 54,500 & 23,700 & -- & 39,100 & 30,700 & 140,000 & 437,000 & 29,500 & 761,000 \\
\hline 1949 & 6,630 & 47,700 & 22,400 & -- & 37,500 & 23,800 & 131,000 & 438,000 & 28,300 & 735,000 \\
\hline 1950 & 7,100 & 56,200 & 26,300 & -- & 45,300 & 32,600 & 151,000 & 501,000 & 31,500 & 851,000 \\
\hline 1951 & 6,860 & 53,600 & 27,700 & -- & 54,000 & 31,700 & 165,000 & 555,000 & 34,600 & 928,000 \\
\hline 1952 & 6,710 & 53,800 & 26,300 & -- & 38,900 & 32,600 & 162,000 & 600,000 & 33,100 & 954,000 \\
\hline 1953 & 6,820 & 53,500 & 28,400 & -- & 54,300 & 31,700 & 170,000 & 605,000 & 34,700 & 985,000 \\
\hline
\end{tabular}


Table 1. Raw materials used in the United States from 1900 through 2006.-Continued

[In thousand metric tons. --, negligible or no data]

\begin{tabular}{|c|c|c|c|c|c|c|c|c|c|c|}
\hline \multirow[b]{2}{*}{ Year } & \multirow[b]{2}{*}{ Agriculture } & \multicolumn{3}{|c|}{ Forestry } & \multicolumn{4}{|c|}{ Metals and minerals } & \multirow[b]{2}{*}{$\begin{array}{c}\text { Nonrenewable } \\
\text { organics }\end{array}$} & \multirow[b]{2}{*}{$\begin{array}{c}\text { Total of } \\
\text { materials }\end{array}$} \\
\hline & & Wood & $\begin{array}{l}\text { Paper and } \\
\text { paperboard }\end{array}$ & $\begin{array}{c}\text { Recycled } \\
\text { paper }\end{array}$ & $\begin{array}{c}\text { Primary } \\
\text { metals }\end{array}$ & $\begin{array}{l}\text { Recycled } \\
\text { metals }\end{array}$ & $\begin{array}{l}\text { Industrial } \\
\text { minerals }\end{array}$ & $\begin{array}{c}\text { Construction } \\
\text { materials }\end{array}$ & & \\
\hline 1954 & 6,680 & 52,900 & 28,500 & -- & 42,200 & 24,600 & 172,000 & 813,000 & 35,300 & $1,180,000$ \\
\hline 1955 & 6,880 & 54,700 & 31,500 & -- & 55,600 & 33,700 & 193,000 & 881,000 & 38,700 & $1,300,000$ \\
\hline 1956 & 6,830 & 55,100 & 33,100 & -- & 49,100 & 38,600 & 201,000 & 942,000 & 41,500 & $1,370,000$ \\
\hline 1957 & 5,990 & 48,700 & 32,000 & -- & 53,400 & 29,500 & 190,000 & 976,000 & 40,900 & $1,380,000$ \\
\hline 1958 & 5,800 & 49,800 & 31,900 & -- & 42,400 & 22,200 & 185,000 & $1,030,000$ & 42,700 & $1,410,000$ \\
\hline 1959 & 6,390 & 54,600 & 35,100 & -- & 51,300 & 27,600 & 211,000 & $1,100,000$ & 46,300 & $1,530,000$ \\
\hline 1960 & 6,380 & 49,100 & 27,200 & 8,400 & 52,800 & 24,900 & 206,000 & $1,120,000$ & 47,300 & $1,540,000$ \\
\hline 1961 & 6,740 & 48,500 & 28,200 & 8,400 & 49,700 & 24,100 & 206,000 & $1,100,000$ & 48,600 & $1,520,000$ \\
\hline 1962 & 7,220 & 50,600 & 30,000 & 8,460 & 55,600 & 24,400 & 217,000 & $1,140,000$ & 51,800 & $1,580,000$ \\
\hline 1963 & 7,400 & 54,200 & 30,700 & 8,970 & 58,200 & 28,300 & 230,000 & $1,270,000$ & 52,800 & $1,740,000$ \\
\hline 1964 & 7,650 & 57,000 & 33,000 & 9,190 & 65,300 & 30,600 & 244,000 & $1,340,000$ & 55,500 & $1,840,000$ \\
\hline 1965 & 7,060 & 60,700 & 31,700 & 9,560 & 75,300 & 34,400 & 258,000 & $1,420,000$ & 59,400 & $1,960,000$ \\
\hline 1966 & 7,540 & 61,000 & 34,900 & 9,880 & 73,600 & 35,300 & 272,000 & $1,480,000$ & 64,000 & $2,030,000$ \\
\hline 1967 & 8,050 & 58,600 & 34,900 & 9,280 & 64,200 & 38,300 & 270,000 & $1,430,000$ & 64,500 & $1,970,000$ \\
\hline 1968 & 9,130 & 60,900 & 37,200 & 9,600 & 78,200 & 38,300 & 280,000 & $1,460,000$ & 70,600 & $2,050,000$ \\
\hline 1969 & 6,890 & 61,000 & 38,200 & 11,200 & 61,600 & 42,000 & 290,000 & $1,520,000$ & 75,700 & $2,100,000$ \\
\hline 1970 & 6,530 & 60,200 & 36,800 & 11,400 & 60,300 & 38,100 & 286,000 & $1,530,000$ & 80,400 & $2,110,000$ \\
\hline 1971 & 6,790 & 64,700 & 37,300 & 11,700 & 64,200 & 38,900 & 289,000 & $1,510,000$ & 81,800 & $2,110,000$ \\
\hline 1972 & 7,590 & 66,900 & 40,500 & 12,400 & 61,900 & 46,600 & 308,000 & $1,540,000$ & 85,400 & $2,170,000$ \\
\hline 1973 & 5,420 & 66,700 & 42,100 & 13,800 & 71,300 & 53,000 & 321,000 & $1,730,000$ & 92,600 & $2,390,000$ \\
\hline 1974 & 4,830 & 57,900 & 40,900 & 14,200 & 63,800 & 57,100 & 315,000 & $1,650,000$ & 91,500 & $2,300,000$ \\
\hline 1975 & 5,770 & 53,500 & 34,900 & 11,900 & 45,600 & 44,100 & 276,000 & $1,420,000$ & 79,900 & $1,980,000$ \\
\hline 1976 & 5,860 & 60,300 & 39,700 & 14,000 & 55,900 & 47,200 & 296,000 & $1,500,000$ & 89,200 & $2,110,000$ \\
\hline 1977 & 5,660 & 66,100 & 40,800 & 14,800 & 63,800 & 46,600 & 313,000 & $1,590,000$ & 97,000 & $2,230,000$ \\
\hline 1978 & 5,410 & 68,800 & 43,100 & 15,200 & 70,700 & 48,300 & 331,000 & $1,730,000$ & 99,100 & $2,410,000$ \\
\hline 1979 & 5,730 & 67,500 & 44,200 & 16,300 & 32,100 & 55,300 & 339,000 & $1,750,000$ & 106,000 & $2,420,000$ \\
\hline 1980 & 5,070 & 58,800 & 42,500 & 16,300 & 48,000 & 49,800 & 311,000 & $1,500,000$ & 112,000 & $2,140,000$ \\
\hline 1981 & 5,140 & 54,700 & 44,000 & 16,100 & 60,500 & 46,600 & 294,000 & $1,330,000$ & 105,000 & $1,960,000$ \\
\hline 1982 & 5,410 & 55,300 & 41,800 & 15,500 & 55,100 & 34,100 & 257,000 & $1,180,000$ & 94,700 & $1,740,000$ \\
\hline 1983 & 5,320 & 66,400 & 46,100 & 17,000 & 46,700 & 40,400 & 274,000 & $1,300,000$ & 93,500 & $1,880,000$ \\
\hline 1984 & 5,740 & 71,500 & 49,500 & 18,600 & 57,900 & 44,000 & 310,000 & $1,480,000$ & 98,700 & $2,130,000$ \\
\hline 1985 & 6,590 & 72,900 & 48,800 & 18,500 & 52,900 & 47,100 & 309,000 & $1,540,000$ & 97,600 & $2,190,000$ \\
\hline 1986 & 6,170 & 77,900 & 50,200 & 20,400 & 46,400 & 47,900 & 299,000 & $1,630,000$ & 102,000 & $2,280,000$ \\
\hline 1987 & 6,480 & 82,700 & 52,700 & 21,800 & 46,000 & 53,000 & 308,000 & $1,800,000$ & 111,000 & $2,480,000$ \\
\hline 1988 & 5,940 & 80,300 & 52,900 & 23,700 & 48,400 & 57,300 & 322,000 & $1,870,000$ & 115,000 & $2,570,000$ \\
\hline 1989 & 5,730 & 81,000 & 51,700 & 24,600 & 43,900 & 55,700 & 318,000 & $1,810,000$ & 114,000 & $2,510,000$ \\
\hline 1990 & 5,530 & 77,900 & 51,300 & 26,400 & 41,900 & 58,900 & 318,000 & $1,840,000$ & 114,000 & $2,540,000$ \\
\hline 1991 & 5,300 & 71,000 & 45,100 & 31,200 & 38,800 & 52,800 & 299,000 & $1,620,000$ & 119,000 & $2,280,000$ \\
\hline 1992 & 5,020 & 74,800 & 48,600 & 30,800 & 44,200 & 54,600 & 309,000 & $1,790,000$ & 125,000 & $2,480,000$ \\
\hline 1993 & 7,590 & 76,200 & 50,400 & 32,200 & 30,100 & 58,700 & 314,000 & $1,890,000$ & 132,000 & $2,590,000$ \\
\hline 1994 & 7,920 & 77,400 & 50,300 & 36,000 & 55,800 & 62,900 & 338,000 & $2,020,000$ & 138,000 & $2,780,000$ \\
\hline 1995 & 5,800 & 77,100 & 48,900 & 38,300 & 54,200 & 64,000 & 341,000 & $2,060,000$ & 135,000 & $2,830,000$ \\
\hline 1996 & 6,160 & 77,800 & 46,400 & 39,100 & 66,000 & 62,200 & 354,000 & $2,140,000$ & 138,000 & $2,930,000$ \\
\hline 1997 & 6,080 & 78,800 & 50,100 & 39,900 & 69,300 & 64,500 & 359,000 & $2,260,000$ & 140,000 & $3,060,000$ \\
\hline 1998 & 5,630 & 80,600 & 50,700 & 40,900 & 79,900 & 61,400 & 368,000 & $2,470,000$ & 135,000 & $3,290,000$ \\
\hline 1999 & 5,330 & 83,100 & 52,700 & 42,500 & 81,900 & 58,600 & 382,000 & $2,530,000$ & 150,000 & $3,390,000$ \\
\hline 2000 & 5,150 & 82,300 & 50,600 & 42,900 & 82,400 & 61,400 & 371,000 & $2,560,000$ & 144,000 & $3,400,000$ \\
\hline 2001 & 4,890 & 80,100 & 45,600 & 42,600 & 65,200 & 59,700 & 363,000 & $2,600,000$ & 135,000 & $3,390,000$ \\
\hline 2002 & 4,650 & 82,600 & 45,000 & 43,200 & 68,000 & 60,700 & 355,000 & $2,520,000$ & 136,000 & $3,320,000$ \\
\hline 2003 & 4,410 & 82,200 & 46,300 & 44,700 & 75,400 & 60,700 & 373,000 & $2,580,000$ & 133,000 & $3,400,000$ \\
\hline 2004 & 4,190 & 90,100 & 48,900 & 45,600 & 85,300 & 63,800 & 391,000 & $2,750,000$ & 141,000 & $3,620,000$ \\
\hline 2005 & 3,980 & 91,400 & 45,900 & 46,500 & 75,000 & 62,800 & 402,000 & $2,850,000$ & 138,000 & $3,720,000$ \\
\hline 2006 & 3,780 & 91,900 & 45,800 & 46,700 & 89,700 & 63,300 & 390,000 & $2,910,000$ & 136,000 & $3,780,000$ \\
\hline
\end{tabular}




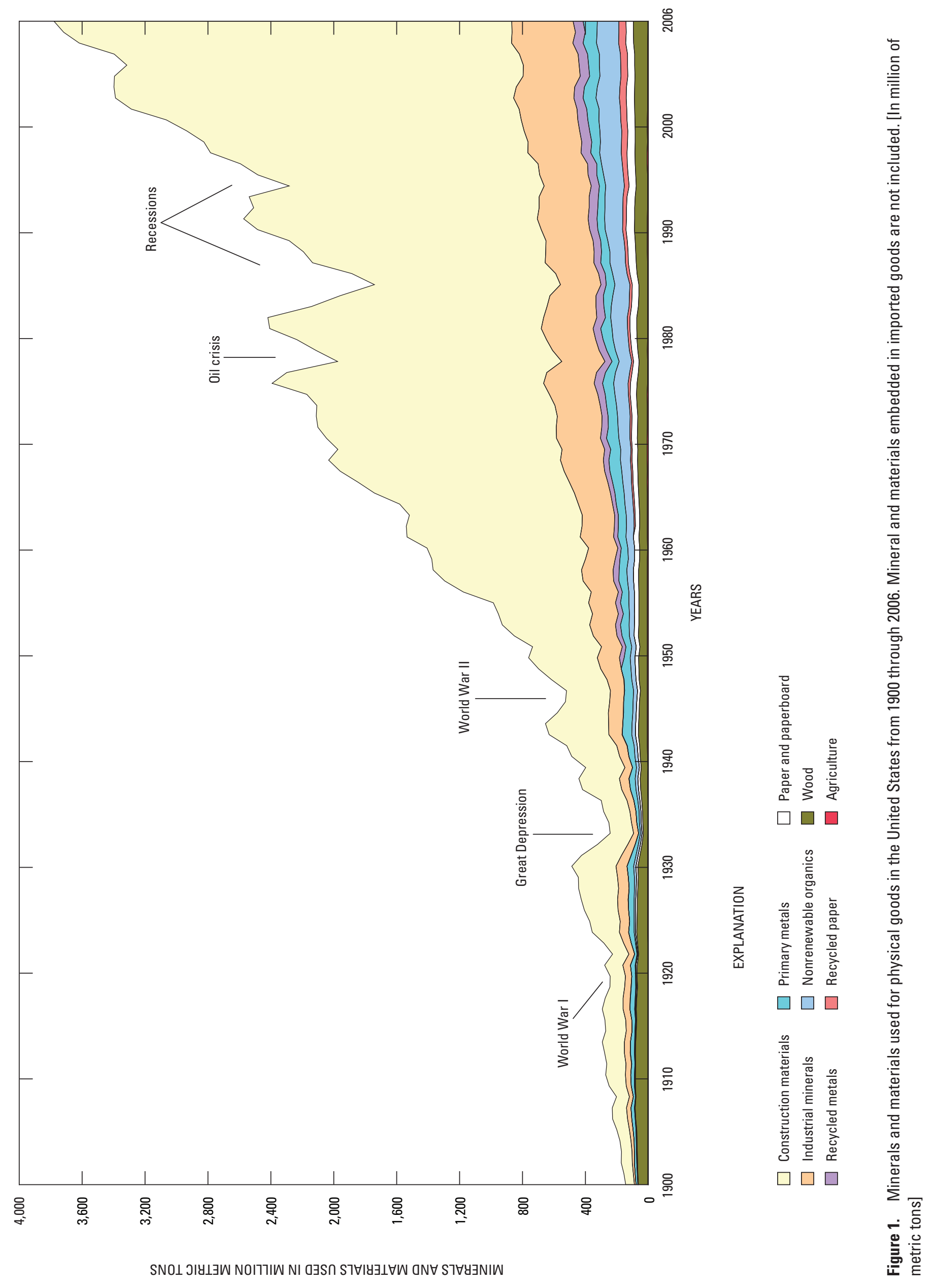




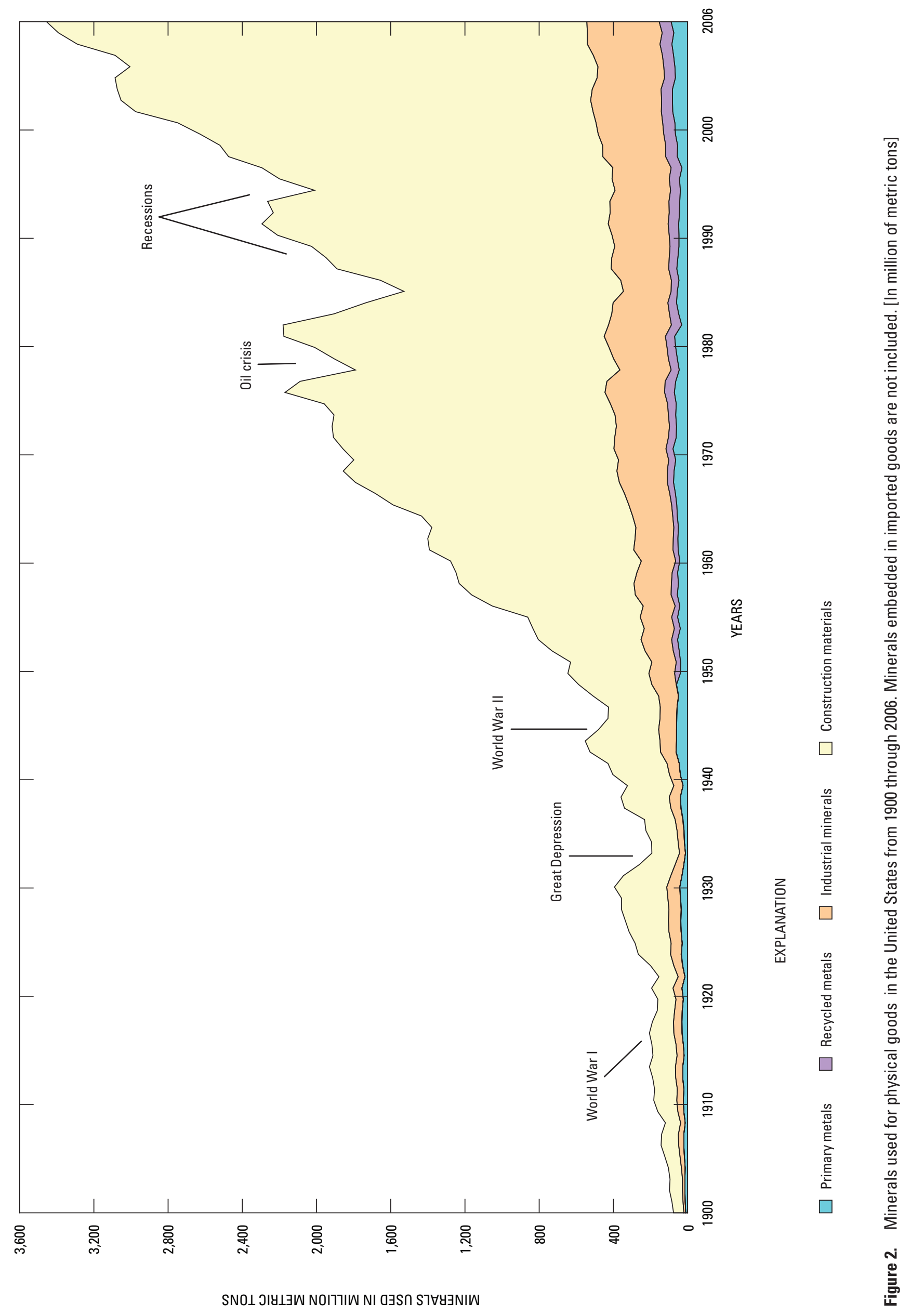




\begin{tabular}{|c|c|c|c|c|}
\hline Agriculture & Forestry & $\begin{array}{c}\text { Metals } \\
\text { (includes recycling) }\end{array}$ & Minerals & Nonrenewable organics \\
\hline $\begin{array}{l}\text { Cotton } \\
\text { Cottonseed } \\
\text { Fishery } \\
\text { Flax seed } \\
\text { Fur } \\
\text { Leather hides } \\
\text { Mohair } \\
\text { Natural rubber } \\
\text { Raw wool } \\
\text { Silk, raw and waste } \\
\text { Tobacco }\end{array}$ & $\begin{array}{l}\text { Paper and paperboard, all grades: } \\
\text { Paper and paperboard, primary: } \\
\text { Insulating board } \\
\text { Hardboard } \\
\text { Wet machine board } \\
\text { Recycled paper, secondary, } \\
\text { 1960-2006: } \\
\text { Recovered paper at paper and } \\
\text { paperboard mills } \\
\text { Recovered paper for other uses } \\
\text { Plywood and veneer } \\
\text { Other forestry: } \\
\text { Cooperage } \\
\text { Fence posts } \\
\text { Hewn ties } \\
\text { Poles and piling } \\
\text { Other miscellaneous products } \\
\text { Wood }\end{array}$ & $\begin{array}{l}\text { Aluminum } \\
\text { Antimony } \\
\text { Arsenic } \\
\text { Beryllium } \\
\text { Bismuth } \\
\text { Cadmium } \\
\text { Cesium } \\
\text { Chromium } \\
\text { Cobalt } \\
\text { Copper } \\
\text { Gallium } \\
\text { Germanium } \\
\text { Gold } \\
\text { Indium } \\
\text { Iron and steel } \\
\text { Lead } \\
\text { Magnesium } \\
\text { Manganese } \\
\text { Mercury } \\
\text { Molybdenum } \\
\text { Nickel } \\
\text { Niobium (columbium) } \\
\text { Platinum group } \\
\text { Rare earths } \\
\text { Rhenium } \\
\text { Selenium } \\
\text { Silicon } \\
\text { Silver } \\
\text { Tantalum } \\
\text { Tellurium } \\
\text { Thallium } \\
\text { Tin } \\
\text { Titanium } \\
\text { Tungsten } \\
\text { Vanadium } \\
\text { Zinc } \\
\text { Tan }\end{array}$ & 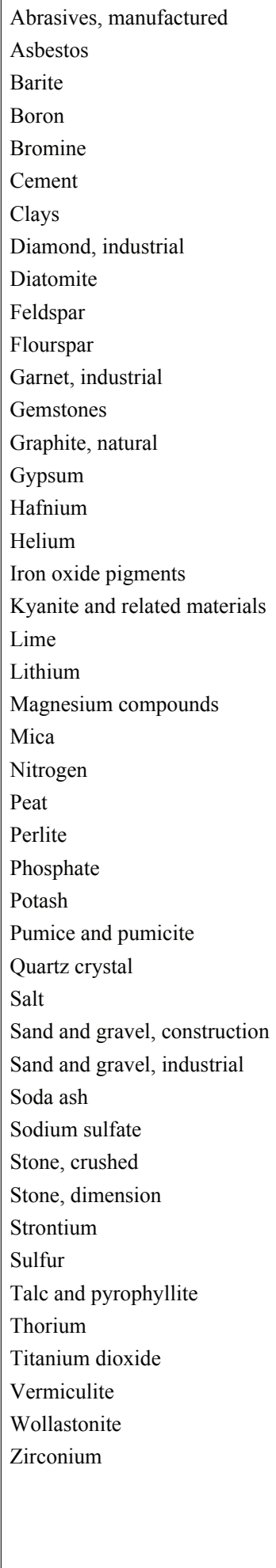 & 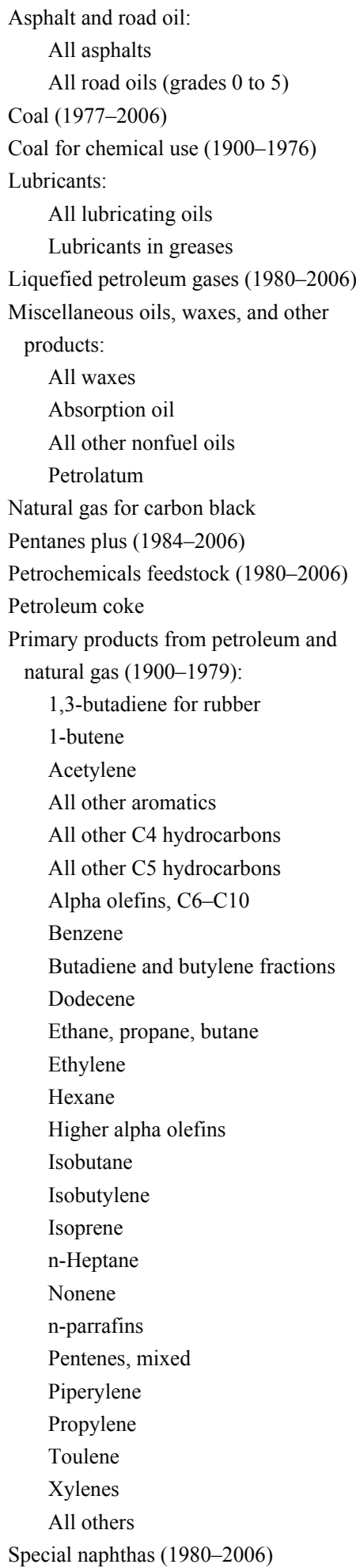 \\
\hline
\end{tabular}

Figure 3. Minerals and materials used in the United States from 1900 through 2006, by category. 